\title{
Optimal Ownership Structures in Asymmetric Joint Ventures
}

\author{
Paul Belleflamme* $\quad$ Francis Bloch ${ }^{\dagger}$
}

April 7, 2000

${ }^{*}$ Department of Economics, Queen Mary \& Westfield College, University of London (UK).

${ }^{\dagger}$ Corresponding author. Department of Economics, Université Catholique de Louvain, 3 Place Montesquieu, 1348 Louvain-la-Neuve (Belgium). Tel: (32) 10473963 or (32) 10 4781 61; fax: (32) 104739 45; e-mail: bloch@ires.ucl.ac.be. We are grateful to Reinhilde Veugelers for helpful discussions. 


\section{Abstract}

This paper investigates the relation between asymmetries in the distribution of shares in joint ventures and asymmetries between the parent companies. When the joint venture and the parent companies are controlled by separate entities, we provide a simple formula to compute the optimal ownership structure. This formula is applied to various models of market interaction, showing that larger companies should have a larger fraction of shares, and so should companies whose goods are closer substitutes of the product of the joint venture, or companies who have a higher cost of transformation of the input produced by a joint venture.

JEL Classification Numbers: D43, L13, L22.

Keywords: joint ventures, strategic alliances, ownership structure, asymmetries. 


\section{Introduction}

Many joint ventures and strategic alliances involve asymmetric partners. In the pharmaceutical industry, new products often result from the collaboration between one large pharmaceutical company and a small researchoriented laboratory $^{1}$. Similarly, in the information technology and space industries, production joint ventures are often concluded between large established companies and small start-up firms which have developed innovative technologies. $^{2}$ In recent years, production joint ventures have been formed by very dissimilar firms belonging to different industries, like the Microcompact car corporation formed by Swatch and Mercedes to market the Smart, Arcor formed by the German railways and a consortium of telecommunications companies to establish a network of cables along German railtracks, or Bluelight.com created by Kmart, the bricks-and-mortar retail giant, and Yahoo!, the online service provider.

In the strategic management literature, the advantages and disadvantages of forming partnerships among asymmetric firms have long been debated: Harrigan (1988) observes that significant asymmetries among sponsoring firms are stabilizing to a venturing relationship (survival and duration) because partners can exploit synergies and complementarities between their assets, but harmful to venturing performance (success) because their hetero-

\footnotetext{
${ }^{1}$ For example, Genentech, a leading biotechnological laboratory has entered a wideranging agreement with the pharmaceutical firm Roche in the early 80's to market its products. In the end, Genentech merged with Roche in 1987.

${ }^{2}$ For example, Microsoft invested in $1997 \$ 45$ million in Lernout \& Hauspie Speech Products, a Belgium-based leading innovator in speech and linguistic technologies, and decided in 1999 to exercise warrants granted under the terms of the first investment to purchase additional shares.
} 
geneity exacerbates differences in how partners value their venture's activities. In the same vein, Doz (1988) suggests that coordination costs may be higher when the joint venture is formed by firms with different backgrounds.

When a joint venture is formed between asymmetric firms, how are the shares of the joint venture distributed among the two partners? The empirical evidence on the ownership structure of asymmetric joint ventures is mixed. On the one hand, as Kesteloot and Veugelers (1996) note, a very large fraction of joint ventures are concluded on the basis of equal shares, even when the partners are asymmetric. (In the sample of joint ventures they study, $50 \%$ of the joint ventures among asymmetric partners involve an equal distribution of shares, and only $11 \%$ a distribution of shares with one dominant partner owning more than $75 \%$ ). On the other hand, anecdotal evidence suggests that in many strategic alliances between asymmetric firms, the dominant partner controls the joint venture. For example, in the pharmaceutical and information technology industries, the large firm typically controls the production of the new product developed in collaboration with the small partner.

In this paper, our goal is to construct a simple theoretical model to study the relation between asymmetry among partners and the distribution of shares in the joint venture. How is the asymmetry in firm's sizes, markets, cost structures reflected in the ownership structure of the joint venture? When is it optimal to adopt an equal sharing rule? When should the firms agree to give all the profits of the joint venture to one of the partners?

In order to answer these questions, we need to distinguish between different sources of asymmetry. First of all, asymmetries can stem from differences in the assets brought by the two companies. If one company provides a larger share of the assets needed by the joint venture, it should be rewarded with a 
higher share of the profits. While this is of course an important determinant of the ownership structure of the joint venture, we do not consider it in the model, and rather focus on asymmetries in the parent companies' costs and market conditions.

The literature on cost-reducing collaborating research , surveyed for example in de Bondt (1997), has analyzed the role of asymmetries on the sizes of firms (Kesteloot and Veugelers (1996), Roller et al. (1998)), on the research spillovers (de Bondt and Henriques (1995)), or on the incentives to form research joint venture and the intensity of research. In this paper, we consider a different model, where the joint venture is formed in order to develop a new product (which can either be marketed or used as an input by the two partners). In this framework, we can explore different sources of asymmetry. We can first study, as in the literature on research joint ventures, the effect of asymmetries in the sizes and cost structures of the two partners. Since the joint venture may be created to launch a new product on the market, we can also analyze asymmetries in the degree of differentiation of the new product with respect to the products of the existing companies. This cannibalization effect of the new product seems to be an important concern in many production joint ventures, and, to the best of our knowledge, has not yet been analytically studied. Finally, when two firms form a joint venture to produce an input, the degree of compatibility of this input with the technologies of the two firms may be different, creating yet another type of asymmetry.

One of the best examples of the relationship between partner asymmetry and ownership structure is the recent joint venture between Mercedes and Swatch. In February 1994, the two companies created a joint venture to produce and market the Smart, a new concept of small urban cars. Mercedes brought to the joint venture its expertise in car making whereas Swatch 
contributed its design and marketing strategies. Notice however that from the start, of the joint venture, the two companies were in very asymmetric positions. In the early 1990's, Mercedes was trying to enter the market for small cars and launching the Class A, a distant competitor to the Smart. On the other hand, Swatch was active in a totally unrelated industry (the watch industry), and had no stakes whatsoever in the automobile industry. Initially, the distribution of shares gave a controlling right to Mercedes, but a $49 \%$ share to Swatch. However, it soon appeared that Swatch was willing to reduce its stake in the joint venture. By August 30, 1997, it only kept $19 \%$ of the shares, and finally sold them to Mercedes on October 31, 1998. ${ }^{3}$

In order to understand how differences in the degree of cannibalization of the new product between the two partners can have led Mercedes to increase its stakes in the joint venture, we construct a very simple model. We suppose that two firms initially earn fixed profits, and can invest in a joint venture to develop a new product or a new input. As in the classical hold-up problem (Williamson (1975)), we suppose that the investments in the joint venture are not observable, and show that, in the second best solution, both firms underinvest with respect to the social optimum. More interestingly, our first result shows that, in order to maximize total profits, the two firms should agree on a distribution of shares which splits equally the surplus generated by the formation of the joint venture. This simple formula can then be applied to different models of asymmetries between firms. In the first model, the two

\footnotetext{
${ }^{3}$ The story of the Smart is in fact very common. A large number of joint ventures are unstable (Kogut (1989)), and end up with one of the partners buying the shares of the other. Noldeke and Schmidt (1998) provide a beautiful explanation for this fact, by showing that option contracts, where one of the partners can buy the shares of the other at a prespecified price implement the first best outcome when investments are sequential.
} 
firms develop a new product and differ in their cost structure. It then turns out that the largest firm (i.e. the firm with the smallest cost) should obtain a higher share in the surplus. In the second model, the two firms differ in the degree of substitutability of their product with respect to the product of the joint venture. In that case, the firm whose product is more highly substitutable to the new product should receive a larger share. In the third model, two firms produce a new input but differ in their ability to transform this input into a final product. It turns out that the firm with the highest cost of transformation of the input should obtain a larger share of the profits of the joint venture.

While the focus of this paper is on the relationship between ownership structure and different types of asymmetries between partners in well structured models of market competition, our work is of course related to the extensive literature on property rights and the resolution of the hold-up problem pioneered by Grossman and Hart (1986) and surveyed in a recent special issue of the Review of Economic Studies (1999). However, in our model, we separate (somewhat artificially) control and ownership rights, by assuming that the joint venture is managed as a separate entity, independent of the parent companies. The ownership rights are then only seen as claims on the profit of the joint venture, and the classical effects of control rights highlighted in Grossman and Hart (1986) and Hart and Moore (1990) are absent from the model.

The rest of the paper is organized as follows. In the next section, we introduce the general problem and characterize the optimal ownership structure. We discuss our three applications to asymmetries in production costs, product differentiation and transformation costs in Section 3. We discuss extensions of the model and open questions for further research in Section 4. 


\section{Optimal ownership structure}

\subsection{The model}

We consider an industry where two firms - the parent companies - initially earn profits $\Pi_{1}^{0}$ and $\Pi_{2}^{0}$ respectively. By investing in research, the two firms can have jointly access to a new activity through a production joint venture. Once the new activity is started, the profits earned respectively by the two parent firms and by the joint venture are given by $\Pi_{1}, \Pi_{2}$ and $\Pi_{3}$. In order to guarantee that the joint venture is profitable, we assume that the total profit earned by the two companies after the start of the new activity is higher than the sum of initial profits. Formally,

Assumption 1. $\Pi_{1}+\Pi_{2}+\Pi_{3}>\Pi_{1}^{0}+\Pi_{2}^{0}$.

We also impose a limitation on transfers between the two companies: they can only transfer money through their participation in the joint venture. Formally,

Assumption 2. $\Pi_{3}>\left|\left(\Pi_{1}-\Pi_{1}^{0}\right)-\left(\Pi_{2}-\Pi_{2}^{0}\right)\right|$.

The probability of success of the new activity is a function of the research investments of the two firms, $a_{1}$ and $a_{2}, p\left(a_{1}, a_{2}\right)$. For simplicity, we assume that the investment levels are bounded and belong to the interval $[0,1]$. We suppose that the investment levels of the two firms are perfect complements and that the probability of success is a symmetric function of the investement levels of the two firms,

$$
p\left(a_{1}, a_{2}\right)=f\left(a_{1} a_{2}\right)
$$


where $f$ is a continuously differentiable,strictly increasing, strictly concave function on $[0,1]$ satisfying $f(0)=0$ and $f(1)=1$.

The profits of the joint venture are distributed according to the shares of the new company owned by the two parents. We let $\alpha$ denote the share of the first company. The expected profit of each firm is thus given by

$$
\begin{aligned}
& \Pi_{1}^{e}=\left[1-f\left(a_{1} a_{2}\right)\right] \Pi_{1}^{0}+f\left(a_{1} a_{2}\right)\left(\Pi_{1}+\alpha \Pi_{3}\right)-a_{1} \\
& \Pi_{2}^{e}=\left[1-f\left(a_{1} a_{2}\right)\right] \Pi_{2}^{0}+f\left(a_{1} a_{2}\right)\left[\Pi_{2}+(1-\alpha) \Pi_{3}\right]-a_{2} .
\end{aligned}
$$

In order to guarantee the existence of a unique interior equilibrium in the model, we make the following additional assumptions on the parameters.

Assumption 3. $\lim _{x \rightarrow 0} x f^{\prime}\left(x^{2}\right)=\infty,\left(\Pi_{1}+\Pi_{2}+\Pi_{3}-\Pi_{1}^{0}-\Pi_{2}^{0}\right) f^{\prime}(1)<1$.

Assumption 4. $-2 x f^{\prime \prime}(x)>f^{\prime}(x)>-x f^{\prime \prime}(x)$.

Assumption 3 is needed to show that the equilibrium level of research investments chosen by the two firms is interior. The first boundary condition implies that the equilibrium investement level is strictly positive, whereas the second boundary condition guarantees that the equilibrium investment level is bounded above by 1 . Assumption 4 is critical to show that the investment levels are strategic complements and that the interior equilibrium level of investment is unique. Notice that, when $\left(\Pi_{1}+\Pi_{2}+\Pi_{3}-\Pi_{1}^{0}-\Pi_{2}^{0}\right)<1$, any function $f(x)=x^{k}$, with $k<1 / 2$ satisfies all our assumptions.

Finally, we suppose that the three firms (the joint venture and the parent companies) are managed as separate, independent entities. The control rights of the joint venture are given to a third party, whose only aim is to maximize the profits of the joint venture. This assumption can often be justified by antitrust considerations, as was clearly the case in the debate surrounding 
the formation of the joint venture between General Motors and Toyota in the mid 1980's. (See Reynolds and Snapp (1986) and Bresnahan and Salop (1986)). Under this assumption of separation of control between the three companies, the profit levels $\Pi_{1}, \Pi_{2}$ and $\Pi_{3}$ are independent of the allocation of shares among the two partners. In the last section of the paper, we discuss alternative control arrangements, where the distribution of shares affects the profits made by the firms.

\subsection{Ownership structure}

We start the analysis by computing the first best solution, when the two firms can perfectly monitor the assets brought in the joint venture, and are able to commit to the investment levels $a_{1}$ and $a_{2}$. The values $a_{1}$ and $a_{2}$ are then selected to maximize

$\Pi_{T}^{e}=\Pi_{1}^{e}+\Pi_{2}^{e}=\left[1-f\left(a_{1} a_{2}\right)\right]\left(\Pi_{1}^{0}+\Pi_{2}^{0}\right)+f\left(a_{1} a_{2}\right)\left(\Pi_{1}+\Pi_{2}+\Pi_{3}\right)-\left(a_{1}+a_{2}\right)$.

In the Appendix, we show that under our assumptions, the first best investment levels are given by $a_{1}=a_{2}=a^{*}$ where $a^{*}$ is implicitly defined by

$$
a^{*} f^{\prime}\left(a^{* 2}\right)\left(\Pi_{1}+\Pi_{2}+\Pi_{3}-\Pi_{1}^{0}-\Pi_{2}^{0}\right)=1 .
$$

The distribution of shares $\alpha$ is computed to ensure that the two firms are willing to participate in the joint venture, i.e., that the following individual rationality constraints hold,

$$
\begin{aligned}
f\left(a_{1} a_{2}\right)\left(\Pi_{1}+\alpha \Pi_{3}-\Pi_{1}^{0}\right)-a_{1} & \geq 0 \\
f\left(a_{1} a_{2}\right)\left(\Pi_{2}+(1-\alpha) \Pi_{3}-\Pi_{2}^{0}\right)-a_{2} & \geq 0 .
\end{aligned}
$$


It is easy to see that, in order to guarantee that the firms participate in the joint venture, the fraction of shares obtained by the first firm $\alpha$ must satisfy

$$
\frac{a^{*}}{f\left(a^{* 2}\right) \Pi_{3}}+\frac{\Pi_{1}^{0}-\Pi_{1}}{\Pi_{3}} \leq \alpha \leq 1-\frac{a^{*}}{f\left(a^{* 2}\right) \Pi_{3}}-\frac{\Pi_{2}^{0}-\Pi_{2}}{\Pi_{3}}
$$

In the second best solution, when investments are not contractible, the time stucture is as follows. First, the two firms sign a contract specifying the distribution of shares $\alpha$. Given the value of $\alpha$, the two firms then select independently their investment levels $a_{1}$ and $a_{2}$. The problem thus becomes

$$
\max \Pi_{T}^{e}=\left[1-f\left(a_{1} a_{2}\right)\right]\left(\Pi_{1}^{0}+\Pi_{2}^{0}\right)+f\left(a_{1} a_{2}\right)\left(\Pi_{1}+\Pi_{2}+\Pi_{3}\right)-\left(a_{1}+a_{2}\right)
$$

subject to

$$
\begin{aligned}
& a_{1} \in \arg \max \Pi_{1}^{e}=\left[1-f\left(a_{1} a_{2}\right)\right] \Pi_{1}^{0}+f\left(a_{1} a_{2}\right)\left(\Pi_{1}+\alpha \Pi_{3}\right)-a_{1}, \\
& a_{2} \in \arg \max \Pi_{2}^{e}=\left[1-f\left(a_{1} a_{2}\right)\right] \Pi_{2}^{0}+f\left(a_{1} a_{2}\right)\left(\Pi_{2}+(1-\alpha) \Pi_{3}\right)-a_{2} .
\end{aligned}
$$

Notice that, given the complementarity of investments, the individual rationality constraints are always satisfied: by selecting $a_{i}=0$, each firm is guaranteed to obtain its initial profit level $\Pi_{i}^{0}$ and hence is always willing to participate in the joint venture.

We now consider, for a fixed value $\alpha$, the equilibrium level of investment chosen by the two firms. We can write

$$
\begin{aligned}
& \frac{\partial \Pi_{1}^{e}}{\partial a_{1}}=a_{2} f^{\prime}\left(a_{1} a_{2}\right)\left(\Pi_{1}+\alpha \Pi_{3}-\Pi_{1}^{0}\right)-1 \\
& \frac{\partial \Pi_{2}^{e}}{\partial a_{2}}=a_{1} f^{\prime}\left(a_{1} a_{2}\right)\left(\Pi_{2}+(1-\alpha) \Pi_{3}-\Pi_{2}^{0}\right)-1
\end{aligned}
$$


By a reasoning similar to the first best case (see the Appendix), it is easy to see that, as long as $\left(\Pi_{1}+\alpha \Pi_{3}-\Pi_{1}^{0}\right)>0$ and $\left(\Pi_{2}+(1-\alpha) \Pi_{3}-\Pi_{1}^{0}\right)>0$, $\frac{\partial^{2} \Pi_{1}^{e}}{\partial a_{1}^{2}}<0, \frac{\partial^{2} \Pi_{2}^{e}}{\partial a_{2}^{2}}<0,\left.\frac{\partial \Pi_{1}^{e}}{\partial a_{1}}\right|_{a_{1}=0}>0,\left.\frac{\partial \Pi_{2}^{e}}{\partial a_{2}}\right|_{a_{2}=0}>0,\left.\frac{\partial \Pi_{1}^{e}}{\partial a_{1}}\right|_{a_{1}=1}<0,\left.\frac{\partial \Pi_{2}^{e}}{\partial a_{2}}\right|_{a_{2}=1}<0$.

Hence, as long as $1-\frac{\Pi_{2}^{0}-\Pi_{2}}{\Pi_{3}} \geq \alpha \geq \frac{\Pi_{1}^{0}-\Pi_{1}}{\Pi_{3}}$, the equilibrium values of $a_{1}$ and $a_{2}$ are given by the solutions to the first order conditions

$$
\begin{aligned}
\frac{\partial \Pi_{1 T}^{e}}{\partial a_{1}} & =a_{2} f^{\prime}\left(a_{1} a_{2}\right)\left(\Pi_{1}+\alpha \Pi_{3}-\Pi_{1}^{0}\right)-1=0, \\
\frac{\partial \Pi_{2}^{e}}{\partial a_{2}} & =a_{1} f^{\prime}\left(a_{1} a_{2}\right)\left(\Pi_{2}+(1-\alpha) \Pi_{3}-\Pi_{2}^{0}\right)-1=0 .
\end{aligned}
$$

Equations(1) and (2) describe the reaction functions in the noncooperative game where the firms choose their investment levels $a_{1}$ and $a_{2}$ when $1-\frac{\Pi_{2}^{0}-\Pi_{2}}{\Pi_{3}} \geq \alpha \geq \frac{\Pi_{1}^{0}-\Pi_{1}}{\Pi_{3}}$. If $\alpha<\frac{\Pi_{1}^{0}-\Pi_{1}}{\Pi_{3}}$ or $1-\frac{\Pi_{2}^{0}-\Pi_{2}}{\Pi_{3}}<\alpha, a_{1} a_{2}=0$ and the new product is not obtained. We conclude that the maximization problem faced by the joint venture can be rewritten as:

$$
\max \Pi_{T}^{e}=\left[1-f\left(a_{1} a_{2}\right)\right]\left(\Pi_{1}^{0}+\Pi_{2}^{0}\right)+f\left(a_{1} a_{2}\right)\left(\Pi_{1}+\Pi_{2}+\Pi_{3}\right)-\left(a_{1}+a_{2}\right)
$$

subject to

$$
\begin{aligned}
& \frac{\partial \Pi_{1}^{e}}{\partial a_{1}}=a_{2} f^{\prime}\left(a_{1} a_{2}\right)\left(\Pi_{1}+\alpha \Pi_{3}-\Pi_{1}^{0}\right)-1=0, \\
& \frac{\partial \Pi_{2}^{e}}{\partial a_{2}}=a_{1} f^{\prime}\left(a_{1} a_{2}\right)\left(\Pi_{2}+(1-\alpha) \Pi_{3}-\Pi_{2}^{0}\right)-1=0, \\
& 1-\frac{\Pi_{2}^{0}-\Pi_{2}}{\Pi_{3}} \geq \alpha \geq \frac{\Pi_{1}^{0}-\Pi_{1}}{\Pi_{3}} .
\end{aligned}
$$

Proposition 1 When investments are not contractible, the optimal distribution of shares is given by

$$
\alpha^{* *}=\frac{1}{2}+\frac{\left(\Pi_{1}^{0}-\Pi_{1}\right)-\left(\Pi_{2}^{0}-\Pi_{2}\right)}{2 \Pi_{3}}
$$


The investment levels are given by $a_{1}=a_{2}=a^{* *}$ where $a^{* *}$ is the solution to

$$
a^{* *} f^{\prime}\left(a^{* * 2}\right)\left(\Pi_{1}+\Pi_{2}+\Pi_{3}-\Pi_{1}^{0}-\Pi_{2}^{0}\right)=2 .
$$

Proof. See the Appendix.

Proposition 1 shows that, when investments are not contractible, the optimal distribution of shares is such that each firm selects the same investment level. Since the assets brought by the two firms are symmetric perfect complements, it is optimal to select a distribution of shares which guarantees that the two firms have the same marginal utility from investing in the joint venture. This leads the two firms to grant a higher share of the joint venture to the firm which suffers most from the creation of the joint venture. In line with the classical result of Grossman and Hart (1986), Proposition 1 also shows that when investments are not contractible, both firms underinvest with respect to the first best. Since investment in the joint venture is a public good for the other firm, each firm selects a lower investment level in the noncooperative case.

\section{Cost asymmetries, product differentiation and optimal ownership structures}

In this section, we illustrate our main result on the optimal distribution of shares, by considering different models of asymmetric firms. In the first two models, we consider the production of a new good which cannibalizes the markets of the two parent companies. Asymmetries between firms are due to differences in the constant marginal cost of production in the first model and to differences in the degree of substitutability of the new product in 
the second model. The third model considers a joint venture producing a common input used by the two firms, as in Rey and Tirole (1999). The two firms differ in the degree of compatibility of their technology with the input produced by the joint venture. In all three models, we suppose that the parent companies and the joint venture are managed as separate entities.

\subsection{Asymmetric production costs}

We model here an industry where two firms initially act as monopolists on their local markets. The new activity consists in the creation of a new product. We consider markets with linear demands, where the two products marketed by the parent companies are independent, and the degree of differentiation of the new product with respect to the two original goods is given by $\gamma$. Hence prices on the market are given by

$$
\begin{aligned}
& P_{1}=1-x_{1}-\gamma x_{3} \\
& P_{2}=1-x_{2}-\gamma x_{3} \\
& P_{3}=1-x_{3}-\gamma\left(x_{1}+x_{2}\right)
\end{aligned}
$$

where $x_{1}, x_{2}$ and $x_{3}$ denote the quantities produced of goods 1,2 and 3, and $0 \leq \gamma \leq 1$ is the degree of differentiation of the new product with respect to the two original products. We suppose that firms have constant marginal cost of production and that the marginal cost of production of firms 2 and 3 is equal to zero, whereas firm 1 has a positive cost of production, $0<c<1$.

We let $a=1-c$, and write the profits of the three firms as

$$
\begin{aligned}
& \Pi_{1}=x_{1}\left(a-x_{1}-\gamma x_{3}\right) \\
& \Pi_{2}=x_{2}\left(1-x_{2}-\gamma x_{3}\right) \\
& \Pi_{3}=x_{3}\left[1-x_{3}-\gamma\left(x_{1}+x_{2}\right)\right] .
\end{aligned}
$$


We consider that the three firms are managed by independent entities, and the three quantities are chosen noncooperatively. Routine computations show that, when the three firms are active on the market, ${ }^{4}$ equilibrium profits are given by

$$
\begin{aligned}
& \Pi_{1}=\left[\frac{a\left(4-\gamma^{2}\right)+\gamma^{2}-2 \gamma}{4\left(2-\gamma^{2}\right)}\right]^{2}, \\
& \Pi_{2}=\left[\frac{a \gamma^{2}+4-\gamma^{2}-2 \gamma}{4\left(2-\gamma^{2}\right)}\right]^{2}, \\
& \Pi_{3}=\left[\frac{2(2-\gamma)-2 a \gamma}{4\left(2-\gamma^{2}\right)}\right]^{2} .
\end{aligned}
$$

Recalling that monopoly profits are given by $\Pi_{1}^{0}=\frac{a^{2}}{4}$ and $\Pi_{2}^{0}=\frac{1}{4}$, we obtain

$$
\alpha^{* *}=\frac{1}{2}-\frac{\gamma(1-a)\left(2-\gamma^{2}\right)}{2(2-\gamma(1+a))} .
$$

Figure 1 depicts the optimal distribution of shares in the parameter space $(a, \gamma)$. When the source of asymmetry are differences in technological efficiency, the most efficient firm (which suffers the highest cost from the introduction of the new product) receives a larger fraction of the shares. Furthermore, by direct differentiation, we obtain the following comparative statics results.

Proposition 2 The fraction of shares of the least efficient firm is decreasing in its cost and increasing in the degree of product differentiation, $\left(\partial \alpha^{* *} / \partial a>\right.$ 0 and $\partial \alpha^{* *} / \partial \gamma<0$.)

\footnotetext{
${ }^{4}$ In order to guarantee that firm one is active on the market, we need to assume: $\gamma^{2}(1-a)-2 \gamma+4 a \geq 0$. Furthermore, assumption 1 is satisfied if and only if $-\gamma^{4}(a+$ $1)^{2}+2 \gamma^{2}\left(3 a^{2}+a+2\right)-16 \gamma(1+a)+8 \geq 0$. It is easily verified that Assumption 2 always holds in this model.
} 


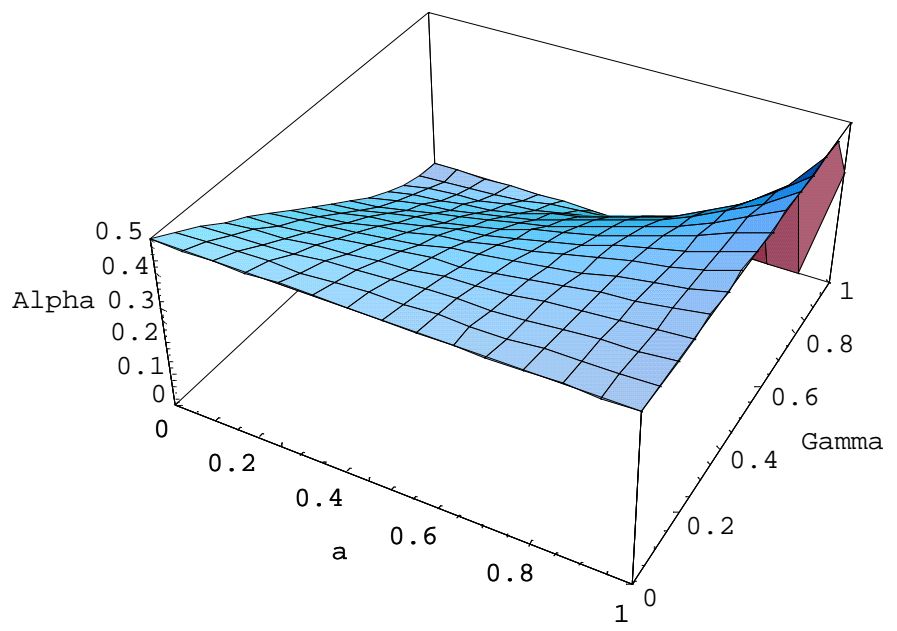

Figure 1: Cost asymmetries

Proof. We compute

$$
\frac{\partial \alpha^{* *}}{\partial a}=\frac{\gamma(1-\gamma)\left(2-\gamma^{2}\right)}{(2-\gamma(1+a))^{2}}>0
$$

and

$$
\frac{\partial \alpha^{* *}}{\partial \gamma}=-\frac{(1-a)\left(2-3 \gamma^{2}+\gamma^{3}(1+a)\right)}{(2-\gamma(1+a))^{2}}<0
$$

Hence, it appears that the distribution of shares is directly related to cost asymmetries between the two firms. The share of the least efficient firm is decreasing with its cost, and converges to $50 \%$ when $a$ goes to 1 . The asymmetry of the ownership structure also disappears as the degree of product differentiation increases. In the limit, when the goods are independent $(\gamma=0)$, the introduction of the new product does not affect the profits of the parent companies, and the optimal distribution of shares is equitable. 


\subsection{Asymmetric product differentiation}

In the second model, we also assume that the new activity consists in the creation of a new product. However, we suppose that the asymmetry between the two firms stems from differences in the degree of substitutability of the new product for the two firms. Formally, we let $\gamma$ denote the degree of differentiation between products 1 and 3 , and $\delta$, the degree of differentiation between products 1 and 2 . The inverse demand schedules are then given by

$$
\begin{aligned}
& P_{1}=1-x_{1}-\gamma x_{3} \\
& P_{2}=1-x_{2}-\delta x_{3} \\
& P_{3}=1-x_{3}-\gamma x_{1}-\delta x_{2} .
\end{aligned}
$$

Assuming that all firms have a zero constant marginal cost, the profit functions are obtained as

$$
\begin{aligned}
& \Pi_{1}=x_{1}\left(1-x_{1}-\gamma x_{3}\right), \\
& \Pi_{2}=x_{2}\left(1-x_{2}-\delta x_{3}\right), \\
& \Pi_{3}=x_{3}\left(1-x_{3}-\gamma x_{1}-\delta x_{2}\right) .
\end{aligned}
$$

With the three firms acting as independent entities, standard computa- 
tions show that equilibrium profits are given $b^{5}$

$$
\begin{aligned}
& \Pi_{1}=\left[\frac{(2-\delta)(2+\delta-\gamma)}{2\left(4-\delta^{2}-\gamma^{2}\right)}\right]^{2} \\
& \Pi_{2}=\left[\frac{(2-\gamma)(2+\gamma-\delta)}{2\left(4-\delta^{2}-\gamma^{2}\right)}\right]^{2} \\
& \Pi_{3}=\left[\frac{2-\gamma-\delta}{4-\delta^{2}-\gamma^{2}}\right]^{2},
\end{aligned}
$$

yielding an optimal ownership structure

$$
\alpha^{* *}=\frac{1}{2}+\frac{(\gamma-\delta)\left(8-\delta^{2}-\gamma^{2}+2 \gamma \delta-2 \delta-2 \gamma\right)}{8(2-\delta-\gamma)} .
$$

It is thus clear that the firm whose product is closest to the new product gets a higher fraction of the shares of the joint venture. Figure 2 graphs the optimal distribution of shares in the parameter space $(\gamma, \delta)$ for values of $\gamma$ and $\delta$ around 0.5 .

The following proposition provides comparative statics on the optimal distribution of shares.

Proposition 3 The optimal fraction of shares is decreasing in the degree of differentiation of one firm's product with the new product, and increasing in the degree of differentiation of the other firm's product, i.e. $\partial \alpha^{* *} / \partial \gamma \geq 0$ and $\partial \alpha^{* *} / \partial \delta \leq 0$.

Proof. We compute

$$
\frac{\partial \alpha^{* *}}{\partial \gamma}=\frac{8-4 \gamma-2 \gamma^{2}+\gamma^{3}-8 \delta+8 \gamma \delta-2 \delta^{2}-3 \gamma \delta^{2}+2 \delta^{3}}{4(2-\gamma-\delta)^{2}}
$$

\footnotetext{
${ }^{5}$ Equilibrium quantities are always positive. Assumption 1 holds provided that 8 $12(\gamma+\delta)+\delta \gamma(\gamma+\delta)+2\left(\gamma^{2}+\delta^{2}\right)+\left(\gamma^{3}+\delta^{3}\right) \geq 0$. Assumption 2 holds provided that $(\gamma-\delta)^{2} \leq \min \{4(1-\gamma) ; 4(1-\delta)\}$. Note that the inequality for Assumption 2 is implied by the inequality for Assumption 1. Whenever the parameters satisfy the first inequality, both assumptions are met.
} 


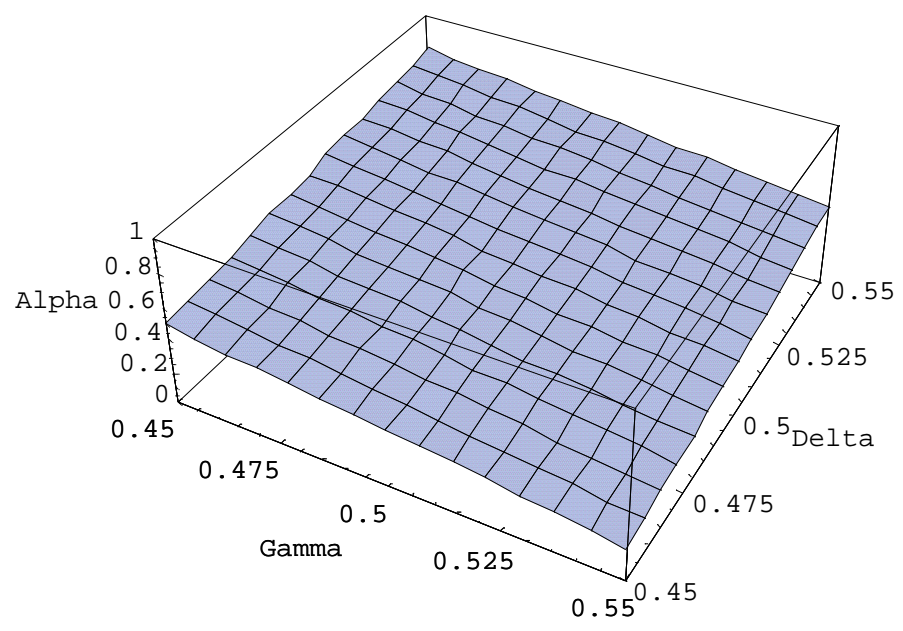

Figure 2: Asymmetries in product differentiation

It is easy to check that the denominator of this expression is decreasing in $\delta$, so that $8-4 \gamma-2 \gamma^{2}+\gamma^{3}-8 \delta+8 \gamma \delta-2 \delta^{2}-3 \gamma \delta^{2}+2 \delta^{3} \geq \gamma(1-\gamma)^{2} \geq 0$. Next observing that $\alpha^{* *}$ is a symmetric function of $\gamma$ and $\delta$ we compute

$$
\frac{\partial \alpha^{* *}}{\partial \delta}=-\frac{\partial \alpha^{* *}}{\partial \gamma} \leq 0
$$

Hence, we observe that the optimal fraction of shares is monotonically related to the degree of substitutability of the new product with respect to the products of the two parents. As the degree of differentiation of the new product with respect to one of the parents increases, the fraction of shares owned by this parent increases, to the detriment of the other parent. 


\subsection{Asymmetric transformation costs}

In the third model, we suppose that a joint venture is created to provide inputs to the two parent companies. The two firms differ in their cost of transforming inputs into the final output. There is no other source of asymmetry between the two firms; in particular, initial profits are assumed to be equal $\left(\Pi_{1}^{0}=\Pi_{2}^{0}\right){ }^{6}$ Let $w$ denote the price of the input as chosen by the joint venture, and let $c_{i}$ denote the extra transformation cost incurred by the parent company $i$. The timing of the game is as follows: in the first stage, the joint venture sets the input price and in the second stage, the parent companies choose the quantities $q_{1}$ and $q_{2}$ of the final output. Demand for the final output is given by the following inverse demand: $p=1-q_{1}-q_{2}$ (we assume $c_{i}<1, i=1,2$ ). We solve the game by backward induction.

Let us first derive the second-stage Cournot-Nash equilibrium. Firm $i$ selects $q_{i}$ to maximize $\Pi_{i}=q_{i}\left(1-w-c_{i}-q_{i}-q_{j}\right)(i, j=1,2, i \neq j)$. Solving for the system of first-order conditions, we get the equilibrium quantities as functions of the input price:

$$
\begin{aligned}
& q_{1}(w)=(1 / 3)\left(1-w-2 c_{1}+c_{2}\right), \\
& q_{2}(w)=(1 / 3)\left(1-w-2 c_{2}+c_{1}\right) .
\end{aligned}
$$

At the first-stage, firm 3 chooses $w$ to maximize

$$
\Pi_{3}=w\left[q_{1}(w)+q_{2}(w)\right]=(w / 3)\left[2(1-w)-c_{1}-c_{2}\right]
$$

yielding

$$
w=\frac{2-c_{1}-c_{2}}{4}
$$

\footnotetext{
${ }^{6}$ We assume moreover that initial profits are such that Assumption 1 is satisfied.
} 
Simple substitutions give the equilibrium profits as ${ }^{7}$

$$
\begin{aligned}
& \Pi_{1}=\left[\frac{2-7 c_{1}+5 c_{2}}{12}\right]^{2} \\
& \Pi_{2}=\left[\frac{2-7 c_{2}+5 c_{1}}{12}\right]^{2} \\
& \Pi_{3}=\frac{\left(2-c_{1}-c_{2}\right)^{2}}{24} .
\end{aligned}
$$

We can now compute the optimal ownership structure as

$$
\alpha^{* *}=\frac{1}{2}+\frac{2\left(c_{1}-c_{2}\right)}{2-c_{1}-c_{2}}
$$

The following proposition provides comparative statics on the optimal distribution of shares.

Proposition 4 The optimal fraction of shares is increasing in the transformation cost of one firm, and decreasing in the transformation cost of the other firm, i.e. $\partial \alpha^{* *} / \partial c_{1}>0$ and $\partial \alpha^{* *} / \partial c_{2}<0$.

Proof. We compute

$$
\frac{\partial \alpha^{* *}}{\partial c_{1}}=\frac{4\left(1-c_{2}\right)}{\left(2-c_{1}-c_{2}\right)^{2}}>0, \text { and } \frac{\partial \alpha^{* *}}{\partial c_{2}}=\frac{-4\left(1-c_{1}\right)}{\left(2-c_{1}-c_{2}\right)^{2}}<0
$$

\section{Extension and conclusion}

Throughout the analysis, we have assumed that the three firms (the parent companies and the joint venture) are managed as separate entities. In particular, the managers of the parent companies were assumed to make their

\footnotetext{
${ }^{7}$ Equilibrium quantities are positive as long as $-2\left(1-c_{2}\right) \leq 5\left(c_{1}-c_{2}\right) \leq 2\left(1-c_{1}\right)$. These conditions also make sure that $0 \leq \alpha^{* *} \leq 1$.
} 
production decisions to maximize profits over their own activity, without taking into account the parents' participation in the joint venture's profits. As a result, the profits $\Pi_{1}, \Pi_{2}$ and $\Pi_{3}$ were independent of the distribution of shares $\alpha$. However, in actual joint ventures, this assumption might very well be violated. In the context of the antitrust investigation over the joint venture between General Motors and Toyota, Bresnahan and Salop (1986) have described a number of control arrangements between the two parent companies, ranging from independence to complete mergers. In many of these arrangements, the objective function of the managers of the three companies depend on the distribution of shares (for example, if the managers of the parent companies take into account their shares of the profit of the joint venture), and our analysis is invalidated.

When the profits of the three companies depend on the distribution of shares, it is impossible to derive explicitly the optimal ownership structure. However, the next proposition shows that this distribution will not correspond to an equal splitting of the surplus, and gives an indication of the direction in which the distribution of shares is biased away from the equal split.

Proposition 5 Suppose that the profits depend on the distribution of shares, and let $\alpha^{* *}$ denote the distribution of shares corresponding to an equal splitting of the surplus. The joint venture has an incentive to increase locally the share $\alpha$ of the first company if and only if total profits $\Pi_{1}+\Pi_{2}+\Pi_{3}$ are increasing in $\alpha$ at $\alpha^{* *}$.

Proof. See the Appendix.

Proposition 5 only gives a partial indication on the optimal ownership structure when profits depend on the distribution of shares, and shows that 
the first firm should obtain a larger fraction of the surplus when total profits are increasing in $\alpha$. It turns out that, even in the simple models presented in Section 3, it is difficult to characterize those situations where total profits are increasing in $\alpha$ at the point of equal splitting of the surplus.

In conclusion, this paper investigates the relation between asymmetries in the distribution of shares in joint ventures and asymmetries between the parent companies. When the joint venture and the parent companies are controlled by separate entities, we provide a simple formula to compute the optimal ownership structure. This formula is applied to various models of market interaction, showing that larger companies should have a larger fraction of shares, companies whose goods are closer substitutes of the product of the joint venture should have a larger fraction of shares, and companies who have a higher cost of transformation of the input produced by a joint venture should have a larger fraction of the shares.

\section{References}

[1] Bresnahan, T. and S. Salop (1986) Quantifying the competitive effects of production joint ventures, International Journal of Industrial Organization 4, 155-175.

[2] De Bondt, R. (1997) Spillover and innovative activities, International Journal of Industrial Organization 15, 1-28.

[3] De Bondt, R. and I. Henriques (1995) Strategic Investment with Asymmetric Spillovers, Canadian Journal of Economics 28, 656-674. 
[4] Doz, Y.(1988) Technology partnerships between larger and smaller firms: Some critical issues, in Cooperative Strategies in International Business, ed. by F. Contractor and P. Lorange. Lexington, MA: Lexington Books.

[5] Grossman, S. and O. Hart (1986) The costs and benefits of ownership: A theory of vertical and lateral integration, Journal of Political Economy 94, 691-719.

[6] Harrigan, K. (1988) Strategic alliances and partner asymmetries, in Cooperative Strategies in International Business, ed. by F. Contractor and P. Lorange. Lexington, MA: Lexington Books.

[7] Hart, O. and J. Moore (1990) Property rights and the nature of the firm, Journal of Political Economy 98, 1119-1158.

[8] Kogut, B. (1989) The stability of joint ventures: Reciprocity and competitive rivalry, Journal of Industrial Economics 38, 183-198.

[9] Noldeke, G. and K. Schmidt (1998) Sequential investments and options to own, RAND Journal of Economics 29, 633-653.

[10] Review of Economic Studies (1999) Special issue on contracts. 66, 1-218.

[11] Rey, P. and J. Tirole (1999) Divergence of objectives and the governance of joint ventures, mimeo., University of Toulouse

[12] Reynolds, R. and B. Snapp (1986) The competitive effects of partial equity interests and joint ventures, International Journal of Industrial Organization 4, 141-153. 
[13] Roller, L. H., M. Tombak and R. Siebert (1998) The incentives to form research joint ventures: Theory and evidence, mimeo., FS IV 98-15, WZB Berlin.

[14] Veugelers, R. and K. Kesteloot (1996) Bargained shares in joint ventures among asymmetric partners: Is the Matthews effect catalyzing? Journal of Economics (Zeitschrift fur Nationalokonomie) 64, 23-51.

[15] Williamson, O. (1975) Markets and Hierarchies: Analysis of Antitrust Implications, New York, NY: Free Press.

\section{Appendix}

\subsection{Determination of the first best solution}

We first show that the investment level $a_{1}=a_{2}=a^{*}$ is the first best choice of the two firms. Deriving total profits with respect to $a_{1}$ and $a_{2}$,

$$
\begin{aligned}
& \frac{\partial \Pi_{T}^{e}}{\partial a_{1}}=a_{2} f^{\prime}\left(a_{1} a_{2}\right)\left(\Pi_{1}+\Pi_{2}+\Pi_{3}-\Pi_{1}^{0}-\Pi_{2}^{0}\right)-1 \\
& \frac{\partial \Pi_{T}^{e}}{\partial a_{2}}=a_{1} f^{\prime}\left(a_{1} a_{2}\right)\left(\Pi_{1}+\Pi_{2}+\Pi_{3}-\Pi_{1}^{0}-\Pi_{2}^{0}\right)-1
\end{aligned}
$$

By concavity of the function $f, \frac{\partial^{2} \Pi_{T}^{e}}{\partial a_{1}^{2}}<0$ and $\frac{\partial^{2} \Pi_{T}^{e}}{\partial a_{2}^{2}}<0$. Now, it is easy to see that, since $\lim _{x \rightarrow 0} x f^{\prime}\left(x^{2}\right)=\infty, \lim _{x \rightarrow 0} f^{\prime}(x)=\infty$. To prove this fact, suppose by contradiction that $\lim _{x \rightarrow 0} f^{\prime}(x)=M$. By continuity of the function $f^{\prime}, \lim _{x \rightarrow 0} f^{\prime}\left(x^{2}\right)=M$, so that $\lim _{x \rightarrow 0} x f^{\prime}\left(x^{2}\right)=0$. Since $\lim _{x \rightarrow 0} f^{\prime}(x)=\infty$,for any positive $a_{2},\left.\frac{\partial \Pi_{T}^{e}}{\partial a_{1}}\right|_{a_{1}=0}>0$. Furthermore, since $f^{\prime}(x)>-x f^{\prime \prime}(x), a_{2} f^{\prime}\left(a_{2}\right)$ is an increasing function. Hence $a_{2} f^{\prime}\left(a_{2}\right)\left(\Pi_{1}+\Pi_{2}+\right.$ $\left.\Pi_{3}-\Pi_{1}^{0}+\Pi_{2}^{0}\right)<f^{\prime}(1)\left(\Pi_{1}+\Pi_{2}+\Pi_{3}-\Pi_{1}^{0}+\Pi_{2}^{0}\right)<1$ so that $\left.\frac{\partial \Pi_{T}^{e}}{\partial a_{1}}\right|_{a_{1}=1}<0$. Hence 
we conclude that the optimal values $a_{1}$ and $a_{2}$ are given by the solutions to the first order conditions

$$
\begin{aligned}
& \frac{\partial \Pi_{T}^{e}}{\partial a_{1}}=a_{2} f^{\prime}\left(a_{1} a_{2}\right)\left(\Pi_{1}+\Pi_{2}+\Pi_{3}-\Pi_{1}^{0}-\Pi_{2}^{0}\right)-1=0, \\
& \frac{\partial \Pi_{T}^{e}}{\partial a_{2}}=a_{1} f^{\prime}\left(a_{1} a_{2}\right)\left(\Pi_{1}+\Pi_{2}+\Pi_{3}-\Pi_{1}^{0}-\Pi_{2}^{0}\right)-1=0 .
\end{aligned}
$$

It is immediate to see that the solution to these equations is symmetric, and $a_{1}=a_{2}=a^{*}$. It remains to check that the individual rationality constraints are satisfied at the first best solution. To this end, we compute the bounds on $\alpha$.

The investment levels are the first best choices, and the bounds on $\alpha$ are computed to make sure that the individual rationality constraints hold. Hence, to finish the computation of the first best solution, it suffices to show that there exist values of $\alpha$ in $[0,1]$ satisfying the inequalities. To this end, it is enough to show that the following three inequalities hold.

$$
\begin{aligned}
\frac{a^{*}}{f\left(a^{* 2}\right) \Pi_{3}}+\frac{\Pi_{1}^{0}-\Pi_{1}}{\Pi_{3}} & \leq 1-\frac{a^{*}}{f\left(a^{* 2}\right) \Pi_{3}}-\frac{\Pi_{2}^{0}-\Pi_{2}}{\Pi_{3}}, \\
1-\frac{a^{*}}{f\left(a^{* 2}\right) \Pi_{3}}-\frac{\Pi_{2}^{0}-\Pi_{2}}{\Pi_{3}} & \geq 0, \\
\frac{a^{*}}{f\left(a^{* 2}\right) \Pi_{3}}+\frac{\Pi_{1}^{0}-\Pi_{1}}{\Pi_{3}} & \leq 1 .
\end{aligned}
$$

The first inequality amounts to $f\left(a^{* 2}\right)\left(\Pi_{1}+\Pi_{2}+\Pi_{3}-\Pi_{1}^{0}-\Pi_{2}^{0}\right)-2 a^{*} \geq 0$, which is always satisfied by optimality of the investment levels. The second and third inequalities are obtained from Assumptions 1 and 2. Notice that these two inequalities can be rewritten as

$$
\begin{aligned}
& f\left(a^{* 2}\right)\left(\Pi_{3}+\Pi_{1}-\Pi_{1}^{0}\right)-a^{*} \geq 0 \\
& f\left(a^{* 2}\right)\left(\Pi_{3}+\Pi_{2}-\Pi_{2}^{0}\right)-a^{*} \geq 0
\end{aligned}
$$


Suppose by contradiction that the first inequality is violated, i.e. $f\left(a^{* 2}\right)\left(\Pi_{3}+\right.$ $\left.\Pi_{1}-\Pi_{1}^{0}\right)-a^{*}<0$. By Assumption 1,

$$
f\left(a^{* 2}\right)\left(\Pi_{1}+\Pi_{2}+\Pi_{3}-\Pi_{1}^{0}-\Pi_{2}^{0}\right)-2 a^{*} \geq 0
$$

so, substracting the two inequalities, we obtain $f\left(a^{* 2}\right)\left(\Pi_{2}-\Pi_{2}^{0}\right)-a^{*}>0$. Hence, $\left(\Pi_{2}-\Pi_{2}^{0}\right)>\left(\Pi_{3}+\Pi_{1}-\Pi_{1}^{0}\right)$, which contradicts Assumption 2. A similar line of reasoning shows that the second inequality is also satisfied.

\subsection{Proof of Proposition 1}

We first show that there exists a unique interior equilibrium in the noncooperative game of choice of investment levels. Using the two reaction functions, we obtain

$$
a_{2}=a_{1} \frac{\left(\Pi_{2}+(1-\alpha) \Pi_{3}-\Pi_{2}^{0}\right)}{\left(\Pi_{1}+\alpha \Pi_{3}-\Pi_{1}^{0}\right)} .
$$

Replacing in equation (2), we have

$$
a_{1} f^{\prime}\left(\frac{\Pi_{2}+(1-\alpha) \Pi_{3}-\Pi_{2}^{0}}{\Pi_{1}+\alpha \Pi_{3}-\Pi_{1}^{0}} a_{1}^{2}\right)\left(\Pi_{2}+(1-\alpha) \Pi_{3}-\Pi_{2}^{0}\right)-1=0 .
$$

Equation (3) provides an implicit definition of the function $\varphi\left(a_{1}\right)$. By Assumption 4, the function $x f^{\prime}\left(c x^{2}\right)$ is decreasing for any positive constant $c$ and any $x \in[0,1]$, so that $\varphi^{\prime}\left(a_{1}\right)<0$. Furthermore, by Assumption 3, we have $\lim _{x \rightarrow 0} x f^{\prime}\left(x^{2}\right)=\infty$, and hence $\varphi(0)>0$. Now,

$$
\varphi(1)=f^{\prime}\left(\frac{\Pi_{2}+(1-\alpha) \Pi_{3}-\Pi_{2}^{m}}{\Pi_{1}+\alpha \Pi_{3}-\Pi_{1}^{0}}\right)\left(\Pi_{2}+(1-\alpha) \Pi_{3}-\Pi_{2}^{m}\right)-1 .
$$

If $\Pi_{2}+(1-\alpha) \Pi_{3}-\Pi_{2}^{0} \geq \Pi_{1}+\alpha \Pi_{3}-\Pi_{1}^{0}$, then $f^{\prime}\left(\frac{\Pi_{2}+(1-\alpha) \Pi_{3}-\Pi_{2}^{m}}{\Pi_{1}+\alpha \Pi_{3}-\Pi_{1}^{0}}\right) \leq f^{\prime}(1)$. Hence, $\varphi(1) \leq f^{\prime}(1)\left(\Pi_{2}+(1-\alpha) \Pi_{3}-\Pi_{2}^{0}\right)-1 \leq 0$. If, on the other hand, 
$\Pi_{2}+(1-\alpha) \Pi_{3}-\Pi_{2}^{0}<\Pi_{1}+\alpha \Pi_{3}-\Pi_{1}^{0}$, note that, by Assumption 4, the function $x f^{\prime}(x)$ is increasing, so that

$$
f^{\prime}\left(\frac{\Pi_{2}+(1-\alpha) \Pi_{3}-\Pi_{2}^{m}}{\Pi_{1}+\alpha \Pi_{3}-\Pi_{1}^{0}}\right) \frac{\Pi_{2}+(1-\alpha) \Pi_{3}-\Pi_{2}^{m}}{\Pi_{1}+\alpha \Pi_{3}-\Pi_{1}^{0}}<f^{\prime}(1) .
$$

In this case, we conclude that $\varphi(1) \leq f^{\prime}(1)\left(\Pi_{1}+\alpha \Pi_{3}-\Pi_{1}^{0}\right)-1 \leq 0$. This argument shows that equation (3) determines the unique equilibrium investment level $a_{1}^{*}$.

Returning to the reaction functions (1) and (2), and totally differentiating them with respect to $\alpha$, we obtain the implicit derivatives

$$
\begin{aligned}
\frac{\partial a_{1}}{\partial \alpha}= & \frac{\Pi_{3} a_{1}}{\left(\Pi_{1}+\alpha \Pi_{3}-\Pi_{1}^{0}\right)\left(\Pi_{2}+(1-\alpha) \Pi_{3}-\Pi_{2}^{0}\right)} \times \\
& \frac{f^{\prime}\left(a_{1} a_{2}\right)\left(\Pi_{1}+\alpha \Pi_{3}-\Pi_{1}^{0}\right)+a_{1} a_{2} f^{\prime \prime}\left(a_{1} a_{2}\right)\left(\Pi_{1}+\Pi_{2}+\Pi_{3}-\Pi_{1}^{0}-\Pi_{2}^{0}\right)}{f^{\prime}\left(a_{1} a_{2}\right)+2 a_{1} a_{2} f^{\prime \prime}\left(a_{1} a_{2}\right)}, \\
\frac{\partial a 2}{\partial \alpha}= & \frac{-\Pi_{3} a_{2}}{\left(\Pi_{1}+\alpha \Pi_{3}-\Pi_{1}^{0}\right)\left(\Pi_{2}+(1-\alpha) \Pi_{3}-\Pi_{2}^{0}\right)} \times \\
& \frac{f^{\prime}\left(a_{1} a_{2}\right)\left(\Pi_{2}+(1-\alpha) \Pi_{3}-\Pi_{2}^{0}\right)+a_{1} a_{2} f^{\prime \prime}\left(a_{1} a_{2}\right)\left(\Pi_{1}+\Pi_{2}+\Pi_{3}-\Pi_{1}^{0}-\Pi_{2}^{0}\right)}{f^{\prime}\left(a_{1} a_{2}\right)+2 a_{1} a_{2} f^{\prime \prime}\left(a_{1} a_{2}\right)} .
\end{aligned}
$$

Now consider the derivative of the profit function with respect to $\alpha$,

$$
\begin{aligned}
\frac{d \Pi_{T}^{e}}{d \alpha}= & {\left[f^{\prime}\left(a_{1} a_{2}\right) a_{2}\left(\Pi_{1}+\Pi_{2}+\Pi_{3}-\Pi_{1}^{0}-\Pi_{2}^{0}\right)-1\right] \frac{\partial a_{1}}{\partial \alpha}+} \\
& {\left[f^{\prime}\left(a_{1} a_{2}\right) a_{1}\left(\Pi_{1}+\Pi_{2}+\Pi_{3}-\Pi_{1}^{0}-\Pi_{2}^{0}\right)-1\right] \frac{\partial a_{2}}{\partial \alpha} } \\
= & f^{\prime}\left(a_{1} a_{2}\right)\left[\left(\Pi_{2}+(1-\alpha) \Pi_{3}-\Pi_{2}^{0}\right) a_{2} \frac{\partial a_{1}}{\partial \alpha}+\left(\Pi_{1}+\alpha \Pi_{3}-\Pi_{1}^{0}\right) a_{1} \frac{\partial a_{2}}{\partial \alpha}\right] \\
= & \frac{a_{1}^{2} a_{2}^{2} f^{\prime}\left(a_{1} a_{2}\right) f^{\prime \prime}\left(a_{1} a_{2}\right)}{f^{\prime}\left(a_{1} a_{2}\right)+2 a_{1} a_{2} f^{\prime \prime}\left(a_{1} a_{2}\right)} \times\left(\Pi_{1}^{0}-\Pi_{1}+\Pi_{3}-\Pi_{2}^{0}+\Pi_{2}-2 \alpha \Pi_{3}\right) \\
& \times \frac{\Pi_{3}\left(\Pi_{1}+\Pi_{2}+\Pi_{3}-\Pi_{1}^{0}-\Pi_{2}^{0}\right)}{\left(\Pi_{1}+\alpha \Pi_{3}-\Pi_{1}^{0}\right)\left(\Pi_{2}+(1-\alpha) \Pi_{3}-\Pi_{2}^{0}\right)} .
\end{aligned}
$$

We have thus established that $\frac{d \Pi_{T}^{e}}{d \alpha} \geq 0$ if and only if $\alpha \leq \frac{1}{2}+\frac{\left(\Pi_{1}^{0}-\Pi_{1}\right)-\left(\Pi_{2}^{0}-\Pi_{2}\right)}{2 \Pi_{3}}$ 
so that the optimal ownership structure is

$$
\alpha^{* *}=\frac{1}{2}+\frac{\left(\Pi_{1}^{0}-\Pi_{1}\right)-\left(\Pi_{2}^{0}-\Pi_{2}\right)}{2 \Pi_{3}} .
$$

It is easy to see that (i) by Assumption 1, $1-\frac{\Pi_{2}^{0}-\Pi_{2}}{\Pi_{3}} \geq \alpha^{* *} \geq \frac{\Pi_{1}^{0}-\Pi_{1}}{\Pi_{3}}$, (ii) by Assumption 2, $1 \geq \alpha^{* *} \geq 0$, and (iii) the equilibrium investment levels for $a=a^{* *}$ are given by $a_{1}=a_{2}=a^{* *}$.

\subsection{Proof of Proposition 5}

In the case where $\Pi_{1}, \Pi_{2}$ and $\Pi_{3}$ depend on the distribution of shares $\alpha$, we obtain the following implicit derivatives when totally differentiating the reaction functions (1) and (2) with respect to $\alpha$ :

$$
\begin{aligned}
\frac{\partial a_{1}}{\partial \alpha}= & \frac{a_{1}^{2} a_{2} f^{\prime \prime}\left(a_{1} a_{2}\right)\left(\Pi_{2}+(1-\alpha) \Pi_{3}-\Pi_{2}^{0}\right)\left(\frac{\partial \Pi_{1}}{\partial \alpha}+\alpha \frac{\partial \Pi_{3}}{\partial \alpha}+\Pi_{3}\right)}{\left[f^{\prime}\left(a_{1} a_{2}\right)+2 a_{1} a_{2} f^{\prime \prime}\left(a_{1} a_{2}\right)\right]\left(\Pi_{1}+\alpha \Pi_{3}-\Pi_{1}^{0}\right)\left(\Pi_{2}+(1-\alpha) \Pi_{3}-\Pi_{2}^{0}\right)}- \\
& \frac{a_{1}\left[a_{1} a_{2} f^{\prime \prime}\left(a_{1} a_{2}\right)+f^{\prime}\left(a_{1} a_{2}\right)\right]\left(\Pi_{1}+\alpha \Pi_{3}-\Pi_{1}^{0}\right)\left(\frac{\partial \Pi_{2}}{\partial \alpha}+(1-\alpha) \frac{\partial \Pi_{3}}{\partial \alpha}-\Pi_{3}\right)}{\left[f^{\prime}\left(a_{1} a_{2}\right)+2 a_{1} a_{2} f^{\prime \prime}\left(a_{1} a_{2}\right)\right]\left(\Pi_{1}+\alpha \Pi_{3}-\Pi_{1}^{0}\right)\left(\Pi_{2}+(1-\alpha) \Pi_{3}-\Pi_{2}^{0}\right)}, \\
\frac{\partial a 2}{\partial \alpha}= & \frac{a_{1} a_{2}^{2} f^{\prime \prime}\left(a_{1} a_{2}\right)\left(\Pi_{1}+\alpha \Pi_{3}-\Pi_{1}^{0}\right)\left(\frac{\partial \Pi_{2}}{\partial \alpha}+(1-\alpha) \frac{\partial \Pi_{3}}{\partial \alpha}-\Pi_{3}\right)}{\left[f^{\prime}\left(a_{1} a_{2}\right)+2 a_{1} a_{2} f^{\prime \prime}\left(a_{1} a_{2}\right)\right]\left(\Pi_{1}+\alpha \Pi_{3}-\Pi_{1}^{0}\right)\left(\Pi_{2}+(1-\alpha) \Pi_{3}-\Pi_{2}^{0}\right)}- \\
& \frac{a_{2}\left[a_{1} a_{2} f^{\prime \prime}\left(a_{1} a_{2}\right)+f^{\prime}\left(a_{1} a_{2}\right)\right]\left(\Pi_{2}+(1-\alpha) \Pi_{3}-\Pi_{2}^{0}\right)\left(\frac{\partial \Pi_{1}}{\partial \alpha}+\alpha \frac{\partial \Pi_{3}}{\partial \alpha}+\Pi_{3}\right)}{\left[f^{\prime}\left(a_{1} a_{2}\right)+2 a_{1} a_{2} f^{\prime \prime}\left(a_{1} a_{2}\right)\right]\left(\Pi_{1}+\alpha \Pi_{3}-\Pi_{1}^{0}\right)\left(\Pi_{2}+(1-\alpha) \Pi_{3}-\Pi_{2}^{0}\right)} .
\end{aligned}
$$


Now reconsider the derivative of the profit function with respect to $\alpha$,

$$
\begin{aligned}
\frac{d \Pi_{T}^{e}}{d \alpha}= & f^{\prime}\left(a_{1} a_{2}\right)\left[\left(\Pi_{2}+(1-\alpha) \Pi_{3}-\Pi_{2}^{0}\right) a_{2} \frac{\partial a_{1}}{\partial \alpha}+\left(\Pi_{1}+\alpha \Pi_{3}-\Pi_{1}^{0}\right) a_{1} \frac{\partial a_{2}}{\partial \alpha}\right] \\
& +f\left(a_{1} a_{2}\right) \frac{\partial\left(\Pi_{1}+\Pi_{2}+\Pi_{3}\right)}{\partial \alpha} \\
= & \left\{\frac{a_{1}^{2} a_{2}^{2} f^{\prime}\left(a_{1} a_{2}\right) f^{\prime \prime}\left(a_{1} a_{2}\right)\left(\Pi_{1}^{0}-\Pi_{1}+\Pi_{3}-\Pi_{2}^{0}+\Pi_{2}-2 \alpha \Pi_{3}\right)}{\left[f^{\prime}\left(a_{1} a_{2}\right)+2 a_{1} a_{2} f^{\prime \prime}\left(a_{1} a_{2}\right)\right]\left(\Pi_{1}+\alpha \Pi_{3}-\Pi_{1}^{0}\right)\left(\Pi_{2}+(1-\alpha) \Pi_{3}-\Pi_{2}^{0}\right)} \times\right. \\
& {\left[\left(\Pi_{2}+(1-\alpha) \Pi_{3}-\Pi_{2}^{0}\right)\left(\frac{\partial \Pi_{1}}{\partial \alpha}+\alpha \frac{\partial \Pi_{3}}{\partial \alpha}+\Pi_{3}\right)-\right.} \\
& \left.\left.\left(\Pi_{1}+\alpha \Pi_{3}-\Pi_{1}^{0}\right)\left(\frac{\partial \Pi_{2}}{\partial \alpha}+(1-\alpha) \frac{\partial \Pi_{3}}{\partial \alpha}-\Pi_{3}\right)\right]\right\} \\
& +\left[f\left(a_{1} a_{2}\right)-\frac{a_{1} a_{2}\left[f^{\prime}\left(a_{1} a_{2}\right)\right]^{2}}{f^{\prime}\left(a_{1} a_{2}\right)+2 a_{1} a_{2} f^{\prime \prime}\left(a_{1} a_{2}\right)}\right] \frac{\partial\left(\Pi_{1}+\Pi_{2}+\Pi_{3}\right)}{\partial \alpha} .
\end{aligned}
$$

Evaluating the previous expression at $\alpha^{* *}=\frac{1}{2}+\frac{\left(\Pi_{1}^{0}-\Pi_{1}\right)-\left(\Pi_{2}^{0}-\Pi_{2}\right)}{2 \Pi_{3}}$, we have

$$
\left.\frac{d \Pi_{T}^{e}}{d \alpha}\right|_{\alpha=\alpha^{* *}}=\left.\left[f\left(a_{1} a_{2}\right)-\frac{a_{1} a_{2}\left[f^{\prime}\left(a_{1} a_{2}\right)\right]^{2}}{f^{\prime}\left(a_{1} a_{2}\right)+2 a_{1} a_{2} f^{\prime \prime}\left(a_{1} a_{2}\right)}\right] \frac{\partial\left(\Pi_{1}+\Pi_{2}+\Pi_{3}\right)}{\partial \alpha}\right|_{\alpha=\alpha^{* *}},
$$

which establishes the result since, according to Assumption 4, the first bracketed term is positive. 